\title{
La prévention du point de vue de ses acteurs
}

\author{
Antoine Casabianca \\ Sezione sanitaria, Dipartimento opere sociali, 6501 Bellinzona
}

Après un bref survol des expériences de prévention connues des participants au groupe de travail - duquel n'étaient pas absents les acteurs sur le terrain - la discussion a permis de mettre en relief les problèmes et les lacunes des programmes actuels et d'entrevoir quelques correctifs

\section{Les difficultés de la prévention}

Une première difficulté est liée aux problèmes de communication entre les différents programmes actuellement en cours en Suisse, la circulation des idées se faisant peu et mal.

D'autre part, cette même communication est freinée par l'absence de dialogue entre des programmes qui se fondent sur des philosophies différentes, les uns étant centrés sur les besoins (présumés) d'une population, les autres sur les facteurs de risque de telle ou telle maladie. Bien qu'il ne soit actuellement pas possible d'indiquer si une approche est meilleure que l'autre l'intégration des deux étant d'ailleurs souvent préconisée - il semblerait que les programmes centrés sur les besoins jouissent d'une moindre publicité surtout du fait que périodiques et congrès scientifiques ne reconnaissent souvent que l'épidémiologie comme base scientifique, au détriment du «social». En outre, à l'intérieur de ces programmes, des modèles sont développés qui en restent souvent peu connus, parce qu'ils ne sont pas (suffisamment) diffusés.

La réalisation des programmes de prévention rencontre souvent des obstacles et des difficultés dont les responsables devraient parler ouvertement; cet échange d'expériences permettant à tous les acteurs une meilleure planification et une allocation des propres ressources plus adéquate en vue d'atteindre les objectifs qui ont été fixés. C'est ainsi que le manque (malheuresement fréquent) de contacts entre programme et bénéficaires - surtout au niveau des entreprises où seul le «chef» sait et décide ce qui est «bon» pour la santé des siens - pourrait trouver une considération plus grande dans les stratégies d'intervention.

Les problèmes politiques que pose l'intervention de l'Etat (légitimité, compétence, stratégies, ...) et les incohérences de la législation actuelle (par exemple: extrême sévérité de certaines normes de protection du paysage et laxisme absolu dans le domaine de l'alimentation) sont rarement discutés ensemble par les responsables des programmes de prévention et une stratégie commune pour les affronter fait encore défaut.

\section{Quelques recommandations}

Les programmes de prévention devraient toujours:

- préciser clairement le type d'approche (philosophie) qu'ils entendent privilégier (besoins/risques; action sociale/action épidémiologique) et tenir compte des particularités régionales;

- chercher à intégrer le plus possible ces deux types d'approches, l'expérience sur le terrain (ligues de la santé, par exemple) pouvant bénéficier des indications des approches scientifiques (PNR 1), mais devant aussi, à son tour, fournir les éléments de l'action sociale indispensables à la re-définition des objectifs et des outils de l'approche de type scientifique;

- faire part de toutes leurs expériences, permettant ainsi le recensement de ce qui se fait actuellement et dans quelles conditions;

- se présenter aux collectivités qui les financent (ligues, communes, cantons, ...) avec des indications claires sur les phases de développement des projets (réflexion, planification, réalisation), sur les ressources disponibles et sur celles qui manquent;

- soumettre leurs résultats à une évaluation permanente - ni seulement économiciste (la prévention rapporte!), ni idéologiquement générique (la santé n'a pas de prix!). 